\title{
Effects of Thromboxane Synthetase Inhibition on Patency and Anastomotic Hyperplasia of Vascular Grafts
}

\author{
Linda M. Graham, M.D., ${ }^{1}$ Thomas E. Brothers, M.D., David Darvisinan, B.S., Karyn A. Harrell, B.A., \\ Christopher K. Vincent, M.D., William E. Burkel, Ph.D., and James C. Stanley, M.D.
}

Departments of Surgery and Anatomy and Cell Biology, University of Michigan Medical Center, Ann Arbor, Michigan 48109; and Department of Surgery, Case Western Reserve University, Cleveland, Ohio 44106

Presented at the Annual Meeting of the Association for Academic Surgery, Salt Lake City, Utah, November 16-19, 1988

\begin{abstract}
The efficacy of a thromboxane synthetase inhibitor (U63,557 A, Upjohn) in promoting early patency and inhibiting anastomotic intimal hyperplasia in ePTFE grafts was compared to that of acetylsalicylic acid (ASA) in a canine model. Animals were started on ASA 5 gr po qd (Group I, $n=12$ ) or U-63,557A $10 \mathrm{mg} / \mathrm{kg}$ po bid (Group II, $n=12) 1$ day before placement of bilateral 5 -mm-i.d., 13- to 16.5-cm-long ePTFE aortoiliac grafts and continued on the medication for the 16-week study. Six dogs in each group received autologous endothelial cell-seeded grafts, while the other six received unseeded grafts. Patency was determined weekly by assessment of femoral pulses. At the conclusion of the study anastomotic intimal hyperplasia was measured on serial sections through the distal anastomosis using a computer-linked digitizer. In Group I the patencies of seeded and unseeded grafts were not significantly different, being 100 and $83 \%$, respectively. Furthermore, luminal narrowing due to intimal hyperplasia was not significantly different being 9.1 $\pm \mathbf{7 . 6 \%}(x \pm \mathrm{SD})$ in seeded grafts and $8.8 \pm \mathbf{8 . 1 \%}$ in unseeded grafts. On the other hand, in Group II the seeded grafts had significantly improved patency when compared to the unseeded grafts $(83 \%$ vs $33 \%, P<0.05)$ and less luminal narrowing $(11.4 \pm 11.1 \%$ vs $21.9 \pm 19.5 \%$, $P<0.01)$. Although $\mathrm{U}-63,557 \mathrm{~A}$ administration promoted patency of unseeded grafts compared to no antiplatelet medication ( $0 \%$ patency), it was significantly less effective than ASA in improving patency $(P<0.05)$ and inhibiting luminal narrowing $(P<0.01)$. ( 1989 Academic Press, Ine.
\end{abstract}

\section{INTRODUCTION}

Research continues to be directed at the development a small caliber prosthetic graft with an acceptable patency rate in peripheral arterial reconstructions. Low patency rates are attributed to early occlusion due to the throm-

\footnotetext{
${ }^{1}$ To whom correspondence should be addressed at Veterans Administration Medical Center, 10701 East Boulevard, Cleveland, OH 44106.
}

bogenicity of the graft surface and late failure due to anastomotic intimal hyperplasia. Many experimental studies have demonstrated the efficacy of pharmacologic agents such as anticoagulant or antiplatelet drugs in promoting early patency. However, the role of antiplatelet agents in promoting long-term patency by reducing anastomotic hyperplasia remains to be clarified.

Endothelial cell (EC) seeding of prosthetic grafts promotes the early development of an endothelial lining and improves the patency of small caliber grafts in experimental animals $[1,6,10,21]$. Such endothelial cell linings have been shown to be functionally intact as evidenced by production of prostacyclin as well as by decreased graft-platelet interactions [4, 22]. Canine studies using large caliber grafts have demonstrated the ability of EC seeding to reduce the midgraft inner capsule thickness compared to unseeded grafts [3]. However, the effect of seeding on anastomotic hyperplasia is uncertain. The usefulness of EC seeding and antiplatelet agents in promoting and reducing anastomotic intimal hyperplasia has been the subject of recent investigations in our laboratory. This report describes the results of studies of anastomotic hyperplasia in EC-seeded and unseeded grafts in animals receiving U-63,557A, a thromboxane synthetase inhibitor which has the theoretical advantage of not inhibiting prostacyclin $\left(\mathrm{PGI}_{2}\right)$ production by the EC. The patency data from this study has been previously published [11]. The "control" animals receiving acetylsalicylic acid (ASA) are part of a multiarm study, part of which has been previously published [12].

\section{MATERIALS AND METHODS}

Patency rate and degree of anastomotic intimal hyperplasia were studied in bilateral aortoiliac expanded polytetrafluoroethylene (ePTFE) grafts, $5 \mathrm{~mm}$ in i.d., 13 to $16.5 \mathrm{~cm}$ long, implanted in adult mongrel dogs weighing 19 to $38 \mathrm{~kg}$. Standard, thin-walled, ePTFE grafts (donated by W. L. Gore and Associates, Flagstaff, AR) were used. These grafts were characterized by nodes of PTFE with longitudinally oriented, interconnecting fibrils having 
nominal lengths of $30 \mu \mathrm{m}$, and a dense, outer circumferential wrap of ePTFE. Two grafts seeded with autologous endothelial cells or two unseeded grafts were implanted in each dog. This procedure was utilized to prevent cross contamination of EC as well as to avoid any adverse systemic effects of one type of graft on the other in dogs receiving both seeded and unseeded prostheses. Dogs were cared for at the Ann Arbor Veterans Administration Medical Center Animal Rescarch Facility which is accredited by the American Association for Accreditation of Laboratory Animal Care. Surgical procedures and animal care complied with the standards in The Guide for the Care and Use of Laboratory Animals (DHFW Publication No. (NIH) 78-23, revised, 1978).

Two different antiplatelet agents were evaluated. Group I dogs $(n=12)$ received ASA 5 gr po qd. Group II animals $(n=12)$ received an oral thromboxane synthetase inhibitor (sodium 5-(3'-pyridinylmethyl)benzofuran-2-carboxylate), U-63,557A (The Upjohn Co., Kalamazoo, MI), $10 \mathrm{mg} / \mathrm{kg}$ po bid. Drug administration began 1 day prior to surgery and continued for the entire 16-week study.

Hematologic and coagulation parameters, including white blood count, platelet count, activated partial thromboplastin time, thrombin time, and fibrinogen levels, were studied in all animals. In addition, platelet aggregation in response to ADP, thrombin, and collagen was measured. Animals were paired on the basis of similarity of these parameters.

Anesthesia was induced with thiamylal sodium and maintained with halothane $/ \mathrm{N}_{2} \mathrm{O} / \mathrm{O}_{2}$ using mechanical ventilation. Penicillin $\mathrm{G}$ benzathine and procaine hydrochloride (450,000 IU each) were administered preoperatively. Animals were hydrated with lactated Ringer's solution $(15 \mathrm{ml} / \mathrm{kg} / \mathrm{hr})$ throughout the operation. A $10-\mathrm{cm}$ segment of external jugular vein was removed for the harvest of autologous endothelial cells. The excised vein was everted over a 5-mm stainless steel rod and incubated for $10 \mathrm{~min}$ at $37^{\circ} \mathrm{C}$ in $0.1 \%$ trypsin in $\mathrm{Ca}^{2+}$ - and $\mathrm{Mg}^{2+}$-free Hanks' balanced salt solution (BSS) containing $0.125 \%$ EDTA at pH 8.0. The vein was then immersed in collagenase (Worthington, CLS Type I, activity $630 \mathrm{U} / \mathrm{ml}$ ) in Hanks' BSS with $\mathrm{Ca}^{2+}$ and $\mathrm{Mg}^{2+}$ at $\mathrm{pH} 7.3$ for $10 \mathrm{~min}$ at $37^{\circ} \mathrm{C}$. A pellet containing an average of $8 \times 10^{5}$ endothelial cells (range, 0.4 to $1.5 \times 10^{6}$ ) was obtained by centrifugation and resuspended in culture medium. While the infrarenal aorta and distal iliac arteries were exposed through a midline abdominal incision, the grafts were filled with autologous blood and incubated for $30 \mathrm{~min}$. After evacuation of this blood, the grafts were flushed with $5 \mathrm{ml}$ of blood containing $1000 \mathrm{IU}$ heparin sodium. Each experimental graft was then filled with half of the EC suspension and allowed to incubate for $15 \mathrm{~min}$, while the graft was rotated every $5 \mathrm{~min}$. Unseeded control grafts underwent identical preparation, except that endothelial cells were not added to the culture medium. After preparation, grafts were placed in a retroperitoneal tunnel between the aorta and external iliac arteries. Animals were systemically anticoagulated with intravenous heparin sodium $(100 \mathrm{IU} / \mathrm{kg})$. After clamping the infrarenal aorta, lateral aortotomies were made and end-to-side anastomoses were created between each graft and the aorta using 6-O polypropylene suture. Care was taken to assure that the second aortotomy corresponded precisely to the first, thereby avoiding asymmetrical anastomotic hemodynamic effects. Clamps were then placed on both distal iliac arteries and an end-to-side anastomosis was completed between each graft and iliac artery using continuous 6-O polypropylene suture. Blood flow was established through both grafts simultaneously. The anticoagulant effect of the heparin was reversed with intravenous administration of protamine sulfate $(1.0 \mathrm{mg} / \mathrm{kg})$. The median sacral artery and the iliac arteries proximal to the distal anastomoses were ligated with $\mathrm{O}$ silk to assure that flow was directed through the grafts. A completion aortogram was performed in all animals to identify and immediately correct any technical problems.

Graft patency was determined by assessment of femoral artery pulses three times a week for the first 2 weeks and weekly thereafter. Patency rates of seeded and unseeded grafts were compared using the $\chi^{2}$ test. The study was continued for a total of 16 weeks except in instances of bilateral graft occlusion after which the prostheses were removed immediately. Weekly serum thromboxane levels were determined for Group II dogs. Immediately prior to the morning dose of U-63,557A, blood samples were drawn into cold syringes containing $0.2 \mathrm{mg}$ ASA. Serum samples were frozen and stored at $70^{\circ} \mathrm{C}$ until radioimmunoassay for the stable metabolite, thromboxane $\mathrm{B}_{2}\left(\mathrm{TXB}_{2}\right)$ [8], was performed at the University of Michigan Medical Center Ligand Laboratory of the Diabetes Research and Training Center.

At the time of sacrifice, animals were anesthetized and the aorta, left renal artery, and iliofemoral arterial systems were isolated. Heparin sodium $(150 \mathrm{IU} / \mathrm{kg})$ was administered intravenously, and an infusion catheter was placed in the left renal artery with outflow cannulae inserted into both femoral arteries. The vessels and grafts were flushed with phosphate-buffered saline solution until the effluent was clear. The infrarenal aorta, aortoiliac grafts, and proximal femoral arteries were then excised. The midsection of one graft was filled with Hanks' BSS and incubated for $5 \mathrm{~min}$ for determination of prostacyclin and thromboxane production. Prostanoid production was similarly measured in a portion of excised distal aorta by radioimmunoassay of the stable degradation products, 6 keto- $\mathrm{PGF}_{1 \alpha}$ and $\mathrm{TXB}_{2}$. Prostanoid production was compared using the unpaired $t$ test. The midportion of each graft was split longitudinally, with one-half being processed for light microscopy and the other half for scanning electron microscopy. Anastomotic regions were fixed in glutaraldehyde, dehydrated in ethanol, embedded in glycol methacrylate, sectioned at $2-\mathrm{mm}$ intervals perpendicular to the long axis of the anastomosis, and stained with methylene blue-basic fuchsin for light microscopic anal- 
ysis (Fig. 1). Using a computer-linked digitizer the circumference of the artery and graft was measured at the intima-media and inner capsule-graft junction allowing calculation of the area of the original lumen. The area of intimal hyperplasia and inner capsule thickening was measured, and the percentage of luminal narrowing was calculated. Anastomotic hyperplasia (intimal hyperplasia of the artery plus inner capsule thickening of the graft) in various groups was compared using the unpaired $t$ test.

\section{RESULTS}

Serum thromboxane levels in Group II animals averaged at least $30 \%$ below preoperative baseline values. This measurement was obtained when serum concentration of U-63,557A was at its trough level.

In seeded grafts of Group I, 12 of $12(100 \%)$ grafts remained patent for the entire 16-week study, while 9 of 12 (75\%) unseeded grafts remained patent (Fig. 2). This approached statistical significance, $P=0.06$. In Group II, 10 of $12(83 \%)$ seeded grafts were patent at 16 weeks, compared to only 4 of $12(33 \%)$ unseeded grafts. This was significantly different, $P<0.05$. In Group I, all occlusions of the unseeded grafts occurred late (after 4 weeks postimplantation) in the study period. However, in Group II one of the occlusions of seeded grafts occurred early (before 4 weeks after implantation), while the other occurred late. In the unseeded grafts of Group II, two occlusions occurred early and the remaining six occurred at or after 4 weeks following graft placement. On macroscopic examination most graft occlusions which occurred after the first 4 weeks appeared to be due to accumulation of tissue at the distal anastomosis.

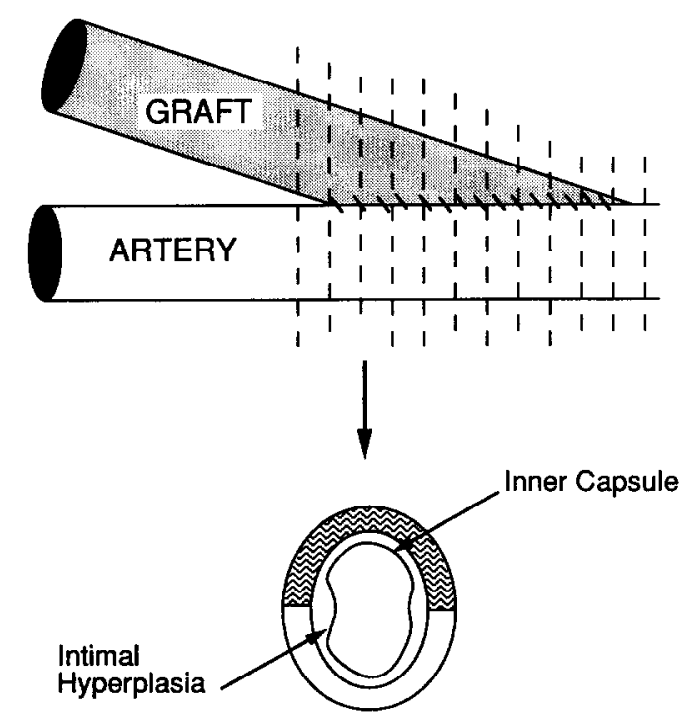

FIG. 1. Serial sections were taken at $2-\mathrm{mm}$ intervals through the distal anastomosis. The area of the inner capsule and intimal hyperplasia was measured using a computer-linked digitizer.

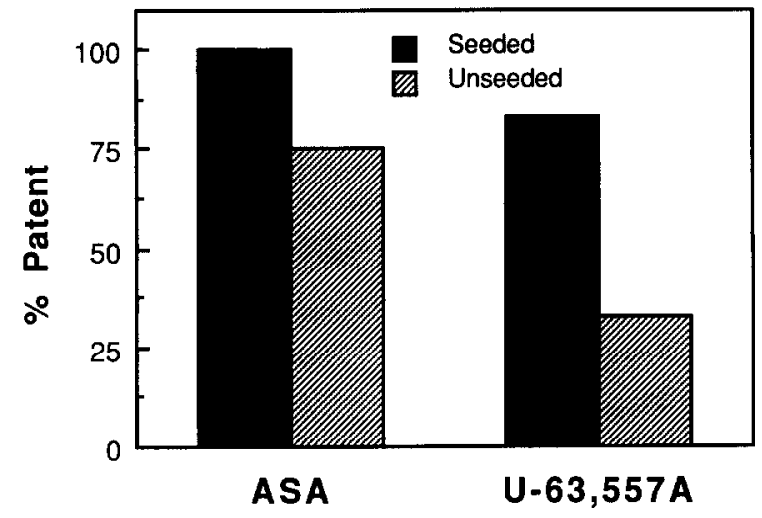

FIG. 2. In dogs receiving ASA the 16 -week patency rate was $100 \%$ in seeded grafts compared to $75 \%$ in unseeded grafts $(P=0.06)$. In animals receiving $U-63,557 \mathrm{~A}$ the 16 -week patency rate was $83 \%$ in seeded grafts compared to $33 \%$ in unseeded grafts $(P<0.05)$.

Luminal production of prostacyclin was significantly greater $(P \leqslant 0.05)$ in seeded grafts than in unseeded grafts of Group I (Table 1). However, both values were markedly less than aortic production. In Group II animals, the difference in $\mathrm{PGI}_{2}$ production between seeded grafts and unseeded grafts did not reach statistical-significance, but again both values were distinctly less than $\mathrm{PGI}_{2}$ production by the aorta. Thromboxane production by seeded grafts in Groups I and II was not significantly different from that in unseeded grafts or aorta.

Histologic studies revealed a cellular lining covering approximately $50 \%$ of the midportion of seeded grafts. This had the appearance of endothelium on scanning electron microscopy (SEM). Cells with the appearance of endothelium were rarely seen away from the anastomotic regions in unseeded grafts, covering less than $10 \%$ of the surface area. Interestingly, in one Group I dog with unseeded grafts, endothelium lined approximately $40 \%$ of each graft.

Measurements of intimal hyperplasia at the distal anastomosis demonstrated no significant difference between seeded and unseeded grafts in Group I (Table 2). Luminal compromise due to the inner capsule of the graft

TABLE 1

Luminal Production of $\mathrm{PGI}_{2}$ and $\mathrm{TXB}_{2}\left(\mathrm{pg} / \mathrm{cm}^{2} / 5 \mathrm{~min}\right)$

\begin{tabular}{|c|c|c|}
\hline & $\mathrm{PGI}_{2}$ & $\mathrm{TXB}_{2}$ \\
\hline \multicolumn{3}{|l|}{ ASA } \\
\hline EC-seeded graft & $70 \pm 50$ & $24 \pm 20$ \\
\hline Unseeded graft & $19 \pm 12$ & $23 \pm 56$ \\
\hline Aorta & $760 \pm 1236$ & $132 \pm 229$ \\
\hline \multicolumn{3}{|l|}{ U-63,557A } \\
\hline EC-seeded graft & $834 \pm 482$ & $1248 \pm 1862$ \\
\hline Unseeded graft & $687 \pm 241$ & $2991 \pm 4282$ \\
\hline Aorta & $4318 \pm 5955$ & $577 \pm 566$ \\
\hline
\end{tabular}

Note. Values are means \pm SDs. 


\section{TABLE 2}

Luminal Narrowing Due to Anastomotic Hyperplasia

\begin{tabular}{lcc}
\hline Treatment & Seeded grafts & Unseeded grafts \\
\hline ASA & $9.1 \pm 7.6 \%$ & $P=8.1 \%$ \\
U-63,557A & $P=0.07$ & $P<0.01$ \\
\hline
\end{tabular}

Note. Values are means $\pm \mathrm{SDs}$

and to intimal hyperplasia at the anastomosis was not significantly different in seeded grafts $(9.1 \pm 7.6 \%)$ compared to that in unseeded grafts $(8.8 \pm 8.1 \%)$. However, in Group II the luminal narrowing in seeded grafts (11.4 $\pm 11.1 \%$ ) was significantly less than that in unseeded grafts $(21.9 \pm 19.5 \%), P<0.01$. When comparing Groups $I$ and II, the difference in luminal narrowing at the anastomoses of seeded grafts approached significance, $P$ $=0.07$, but in unseeded grafts was clearly significantly less, $P<0.01$, in Group I.

\section{DISCUSSION}

This investigation was undertaken to evaluate the effect of various antiplatelet drugs and $\mathrm{EC}$ seeding on prevention of graft failure due to early thrombosis and later development of anastomotic intimal hyperplasia. The aortoiliac model was selected to allow study of hyperplasia at an end-to-side anastomosis. Previous studies in our laboratories had demonstrated that the patencies of seeded and unseeded small caliber grafts were similar if no antiplatelet agents were utilized to inhibit thrombosis until an endothelial cell surface had developed [10, 21]. However, with the perioperative use of acetylsalicylic acid and dipyridamole, EC seeding significantly improved that 16 week patency rate of 4-mm-i.d. ePTFE aortoiliac grafts from $0 \%$ for control grafts to $50 \%$ for seeded grafts [10]. Since evaluation of anastomotic hyperplasia requires the patency of some control grafts, 5-mm ePTFE grafts were used in the present study.

A thromboxane synthetase inhibitor was selected for comparison with ASA with the expectation that continued $\mathrm{PGI}_{2}$ production by EC-seeded grafts would prove beneficial in promoting long-term patency. As anticipated, $\mathrm{PGI}_{2}$ production by the graft and aortic surface was greater in U-63,557A animals than in ASA-treated dogs. However, TXB 2 production was also greater in U-63,557A animals. The reason for this is uncertain and may be a reflection of the short half-life of U-63,557A since dogs were sacrificed 15 to $20 \mathrm{hr}$ after their last dose of the inhibitor. However, this may also be an indication of suboptimal levels of U-63,557A. Cellular thromboxane biosynthesis is inhibited in a dose-dependent fashion, and the dose selected has been shown to maintain plasma concentrations U-63,557A above $0.5 \mu \mathrm{g} / \mathrm{ml}$ for $12 \mathrm{hr}$ [23]. Serum thromboxane levels drawn immediately prior to the animal's next dose of $\mathrm{U}-63,557 \mathrm{~A}$ averaged $30 \%$ below baseline. Both Freeman et al. [10] and McDaniel et al. [15] found a greater decrease in thromboxane levels after treatment with $\mathrm{U}-63,557 \mathrm{~A} 10 \mathrm{mg} / \mathrm{kg}$ administered three times a day rather than twice a day as in the present study, but the time of blood sampling was not reported.

The aim of the present study was to evaluate the effectiveness of various agents in promoting early graft patency. Since our previous studies demonstrated $0 \% 2$-week patency of 5-mm-i.d. ePTFE aortoiliac grafts without the use of antiplatelet agents, both ASA and U-63,557A promoted the patency of unseeded grafts in the current study. These results confirm and extend those of Endean et al. [7] who studied vein grafts and McDaniel et al. [15] who showed improved 1 week patency of 4-mm-i.d., 4-cm-long Dacron and ePTFE grafts implanted in the carotid artery of dogs receiving U-63,557A or ASA-dipyridamole compared to no medication. However, neither Schmidt and colleagues [19] nor Freeman et al. [9] found significant improvement in the patency of 4-mm-i.d., 6-cm-long Dacron or ePTFE grafts in dogs treated with U-63,557A compared to no medication in studies extending 4 to 5 weeks, perhaps a reflection of the longer grafts utilized and the longer period of study. In addition, in our studies, as well as in those of others $[9,19,20], \mathrm{U}-63,557 \mathrm{~A}$ was not as effective as ASA in promoting the patency of unseeded grafts. Since the effectiveness of antiplatelet agents in maintaining short-term graft patency may be related to decreased early platelet deposition [2], the reduced effectiveness of the thromboxane synthetase inhibitor may be due to the accumulation of proaggregatory endoperoxide intermediates $\mathrm{PGG}_{2}$ and $\mathrm{PGH}_{2}[14,18,23]$. Interestingly, Sharp et al. [20] noted a significant improvement in the patency of EC-seeded Dacron grafts in dogs receiving U-63,557A compared to unmedicated animals, but no difference between these groups when studying unseeded grafts. Thus, U-63,557A appears to be capable of promoting graft patency until sufficient EC are present to maintain graft patency.

Most graft failures in our study occurred at or after 1month postimplantation, and appeared to be secondary to intimal hyperplasia at the anastomosis. However, the causative factors in anastomotic hyperplasia and particularly the relationship between early platelet deposition and the development of anastomotic hyperplasia are not clearly defined [5]. Although many studies have investigated the efficacy of antiplatelet agents in promoting patency, few studies have assessed their effect on intimal hyperplasia of grafts. Our study as well as others suggests that ASA with or without dipyridamole may inhibit intimal hyperplasia [9, 16, 17]. Studies in our laboratory have suggested that ASA is more effective than other antiplatelet drugs and even other cyclooxygenase inhibitors in controlling anastomotic hyperplasia [12]. However, the 
method by which ASA reduces intimal thickening is unknown. Hansen et al. [13] have shown that both ASAdipyridamole and ticlopidine (an antiaggregating agent) reduce platelet deposition on ePTFE grafts, but only ASA-dipyridamole decreases intimal hyperplasia and improves 3 -month patency rates. This suggests that the benefit of ASA is not simply due to inhibition of platelet deposition.

Long-term graft patency requires controlling tissue accumulation at the anastomoses and in downstream vessels. Although anastomotic hyperplasia was similar in seeded and unseeded grafts in ASA-treated dogs, anastomotic intimal hyperplasia was significantly reduced in seeded grafts compared to that in unseeded grafts in U63,557A-treated animals. This suggests that under certain circumstances, the presence of an endothelial surface in the graft might contribute to the control of anastomotic hyperplasia. The nature of these circumstances and the mechanism of such control remain to be elucidated.

\section{ACKNOWLEDGMENTS}

This research was supported by grants from the Veterans Administration and the Upjohn Co.

\section{REFERENCES}

1. Allen, B. T., Long, J. A., Clark, R. E., Sicard, G. A., Hopkins, $K$. T., and Welch, M. J. Influence of endothelial cell seeding on platelet deposition and patency in small-diameter Dacron arterial grafts. J. Vasc. Surg. 1: 224, 1984.

2. Allen, B. T., Sicard, G. A., Welch, M. J., Mathias, C. J., and Clark, R. E. Platelet deposition on vascular grafts. The accuracy of in vivo quantitation and the significance of in vivo platelet reactivity. Platelet deposition on vascular grafts. Ann. Surg. 203: 318, 1986.

3. Burkel, W. E., Ford, J. W., Vinter, D. W., Kahn, R. H., Graham, L. M., and Stanley, J. C. Fate of knitted Dacron velour vascular grafts seeded with enzymatically derived autologous canine endothelium. Trans. Amer. Soc. Artif. Intern. Org. 28: 178, 1982.

4. Clagett, G. P., Burkel, W. E., Sharefkin, J. B., Ford, J. W., Hufnagel, H., Vinter, D. W., Kahn, R. H., Graham, L. M., Stanley, J. C., and Ramwell, P. W. Platelet reactivity in vivo in dogs with arterial prostheses seeded with endothelial cells. Circulation 69: 632, 1984.

5. Clowes, A. W. The role of aspirin in enhancing arterial graft patency. J. Vasc. Surg. 3: 381, 1986.

6. Douville, E. C., Kempczinski, R. F., Birinyi, L. K., and Ramalanjaona, G. R. Impact of endothelial cell seeding on long-term patency and subendothelial proliferation in a small-caliber highly porous polytetrafluoroethylene graft. J. Vasc. Surg. 5: 544, 1987.

7. Endean, E. D., Boorstein, J. M., Hees, P. L., and Cronewett, J. L. Effect of thromboxane synthetase inhibition on canine autogenous vein grafts. J. Surg. Res. 4: 297, 1986.

8. Fitzpatrick, F. A., Gorman, R. R., McGuire, J. C., Kelly, R. C., Wynalda, M. A., and Sun, F. F. A radioimmunoassay for thromboxane $\mathrm{B}_{2}$. Anal. Biochem. 82: 1, 1977.

9. Freeman, M. B., Sicard, G. A., Valentin, L. I., Neuberger, S., Mangino, M. J., and Anderson, C. B. The association of in vitro arachidonic acid responsiveness and plasma thromboxane levels with early platelet deposition on the luminal surface of small-diameter grafts. J. Vasc. Surg. 7: 554, 1988.

10. Graham, L. M., Stanley, J. C., and Burkel, W. E. Improved patency of endothelial cell seeded long, $4 \mathrm{~mm}$ diameter knitted Dacron and ePTFE vascular prostheses. ASAIO J. 8: 65, 1985.

11. Graham, L. M., Stanley, J. C., and Burkel, W. E. Influence of endothelial cell seeding and antiplatelet drugs on patency of prosthetic vascular grafts. In P. P. Zilla, R. D. Fasol, and M. Deutsch (Eds.), Endothelialization of Vascular Grafts. Basel: Kargar, 1987. p. 57.

12. Graham, L. M., Vincent, C. K., Brothers, T. E., Harrell, K. A., Darvishian, D., Sell, R., Burkel, W. E., and Stanley, J. C. Efficacy of antiplatelet agents in promoting patency and reducing anastomotic hyperplasia of endothelial cell (EC) seeded and unseeded ePTFE grafts. Surg. Forum 39: 348, 1988.

13. Hansen, K. J., Howe, H. R., Edgerton, T. A., Faust, K. B., Kon, N. D., Geisinger, K. R., and Meredith, J. H. Ticlopidine versus aspirin and dipyridamole: Influence on platelet deposition and three-month patency of polytetrafluoroethylene grafts. J. Vasc. Surg. 4: 174, 1986.

14. Jones, E. W., Cockbill, A. J., Cowley, A. J., Hanley, S. P., and Heptinstall, S. Effects of dazoxiben and low-dose aspirin on platelet behaviour in man. Brit. J. Clin. Pharmacol. 15: 39S, 1983.

15. McDaniel, M. D., Huntsman, T., Miett, T. O'C., and Cronenwett, J. L. Effect of a selective thromboxane synthetase inhibitor on arterial graft patency and platelet deposition in dogs. Arch. Surg. 122: 887, 1987.

16. Metke, M. P., Lie, J. T., Fuster, V., Josa, M., and Kaye, M. P. Reduction of intimal thickening in canine coronary bypass vein grafts with dipyridamole and aspirin. Amer. J. Cardiol. 43: 1144, 1979.

17. Oblath, R. W., Buckley, F. O., Jr., Green, R. M., Schwartz, S. I., and DeWeese, J. A. Prevention of platelet aggregation and adherence to prosthetic vascular grafts by aspirin and dipyridamole. Surgery 84: 37, 1978.

18. Reilly, I. A. G., Doran, J. B., Smith, B., and FitzGerald, G. A. Increased thromboxane biosynthesis in a human preparation of platelet activation: Biochemical and functional consequences of selective inhibition of thromboxane synthase. Circulation 73: 1300, 1986.

19. Schmidt, S. P., Hunter, T. J., Falkow, L. J., Evancho, M. M., and Sharp, W. V. Effects of antiplatelet agents in combination with endothelial cell seeding on small-diameter Dacron vascular graft performance in the canine carotid artery model. J. Vasc. Surg. 2: $898,1985$.

20. Sharp, W. V., Schmidt, S. P., and Donovan, D. L. Prostaglandin biochemistry of seeded endothelial cells on Dacron prostheses. $J$. Vasc. Surg. 3: 256, 1986.

21. Stanley, J. C., Burkel, W. E., Ford, J. W., Vinter, D. W., Kahn, R. H., Whitehouse, W. M., Jr., and Graham, L. M. Enhanced patency in endothelial cell seeded small diameter externally supported Dacron iliofemoral interposition grafts. Surgery 92: 994, 1982.

22. Whitehouse, W. M., Wakefield, T. W., Vinter, D. W., Ford, J. W. Swanson, D. P., Thrahl, J. H., Froelich, J. W., Brown, L. E., Burkel, W. E., Graham, L. M., and Stanley, J. C. Indium-111-oxine labeled platelet imaging of endothelial seeded Dacron thoracoabdominal vascular prostheses in a canine model. Trans. Amer. Soc. Artif. Intern. Org. 29: 183, 1983.

23. Wynalda, M. A., Liggett, W. F., and Fitzpatrick, F. A. Sodium 5(3'-pyridinylmethyl)benzofuran-2-carboxylate (U-63557A), a new, selective thromboxane synthase inhibitor: Intravenous and oral pharmacokinetics in dogs and correlations with ex situ thromboxane $\mathrm{B}_{2}$ production. Prostaglandins 26: 311, 1983. 